\title{
Le « patrimoine martyr »
}

Résurrection des monuments historiques en Europe après 1945

The Restoration of "Martyred Heritage" in Europe after 1945

\section{Nicolas Detry}

\section{(2) OpenEdition}

Journals

Édition électronique

URL : http://journals.openedition.org/crau/395

DOI : $10.4000 /$ crau.395

ISSN : 2547-5746

Éditeur

Éditions du patrimoine

Édition imprimée

Date de publication : 1 décembre 2014

Pagination : 67-89

ISBN : 978-2-7577-0379-3

ISSN : 1296-4077

Référence électronique

Nicolas Detry, «Le " patrimoine martyr » », Les Cahiers de la recherche architecturale et urbaine [En ligne], 30/31 | 2014, mis en ligne le 17 septembre 2017, consulté le 03 mai 2019. URL : http:// journals.openedition.org/crau/395; DOI : 10.4000/crau.395 


\section{Le « patrimoine martyr » \\ Résurrection des monuments historiques en Europe après 1945}

\section{La restauration des monuments historiques en Europe à l'issue de la Seconde Guerre mondiale Méthodes, théories et pratiques}

Sur le temps long, l'histoire de l'architecture se déroule en parallèle avec une succession de guerres, de violences et de destructions. Avant le XIX siècle, l'évolution de la restauration a été efficacement définie comme " architecture sur le préexistant diversement évalué dans le temps ${ }^{1} »$. En effet, dans l'antiquité plutôt que détruire les temples et les palais « ennemis » on les réutilisait en les adaptant. Les temples païens devenaient des églises, les églises devenaient des mosquées puis à nouveau des églises. Mais au Xx siècle, "le siècle court ${ }^{2}$ ", l'histoire prend un coup d'accélérateur inouï. C'est alors que l'on peut parler de "patrimoine martyr ", phénomène qui a pris de l'ampleur dans des guerres récentes (ex-Yougoslavie par exemple), qui ne sont plus nécessairement des guerres de conquêtes, mais des guerres internes, des guerres ethniques, dites aussi guerres « civiles », ayant souvent des conflits religieux en arrière-fond. Le $\mathrm{xx}^{\mathrm{e}}$ siècle est meurtri par trois types

1. Guglielmo de Angelis d'Ossat,

«Restauro come architettura sulle preesistenze diversamente valutate nel tempo », Palladio, Rome, 1978.

2. En référence au livre de Eric J. Hobsbawm, L'Âge des extrêmes, histoire du court $x x^{e}$ siècle, (éd. anglaise 1994), Paris/Bruxelles, André Versailles éditeur/Le Monde diplomatique, 2008. 


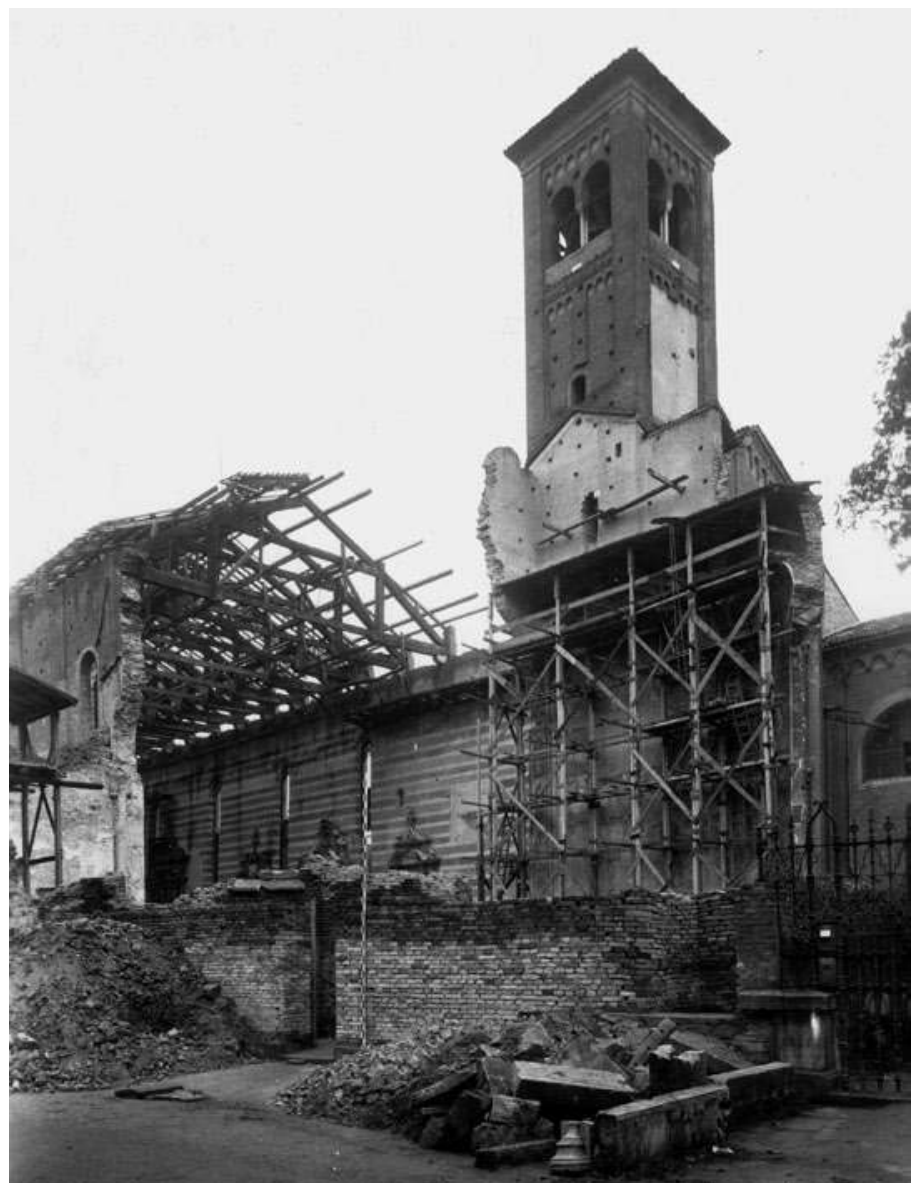

Padoue, chevet de

I'église degli Eremitani, en 1945 , début des

travaux de restauration.

Sources archives:

MIBACT-SBAP-VeBPT-AF,

Archivio Fotografico.

Padoue, intérieur de l'église degli Eremitani, vers 1935 .

Sources archives: MIBACT-SBAP-VeBPT-AF Archivio Fotografico.

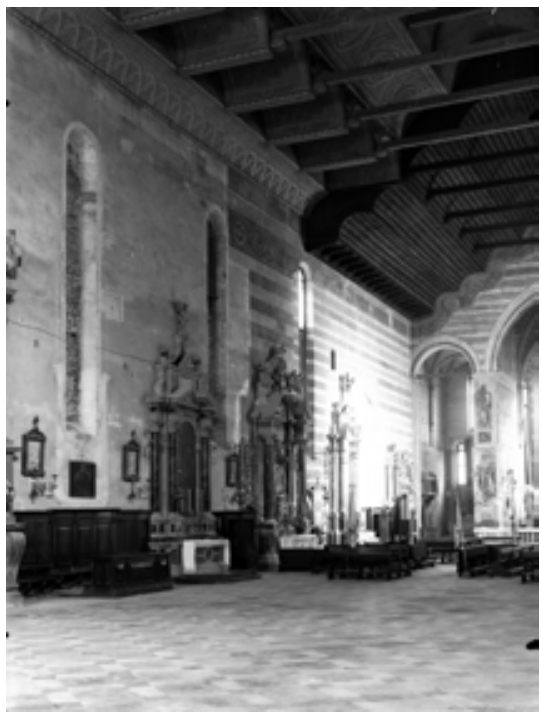

Padoue, façade Sud de l'église degli Eremitani, dessin de la Sovrintendenza (début du $\mathrm{xx}^{\mathrm{e}}$ siècle ?) en vue de réaliser des travaux. À l'extrémité droite (à l'Est) la Chapelle Ovetari qui fut détruite en 1944 . Sources archives: MIBACT-SBAPVeBPT-AF, Archivio Fotografico.

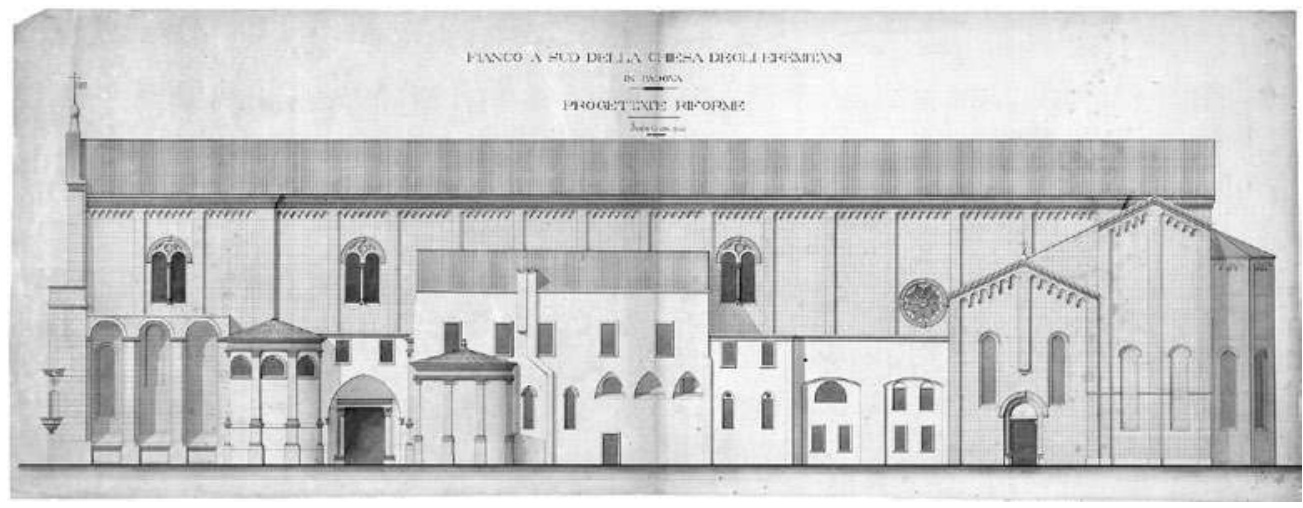


de guerre: les deux guerres mondiales, la Guerre froide, et les guerres dites « civiles», conséquences de la décolonisation et de l'éclatement du bloc soviétique. Ce dernier fait majeur (novembre 1989) marque la fin de la bipolarisation du monde (États-Unis-URSS); d'où la résurgence, à la fin du $x x^{e}$ siècle et au début du $x \times\left.\right|^{e}$ siècle, de diverses formes de nationalismes, d'intégrismes religieux ou ethniques, accompagnés de conflits territoriaux. Les biens culturels, otages de ces phénomènes sociétaux, peuvent devenir " patrimoine martyr ». II s'agit notamment de l'architecture prise comme cible dans les conflits armés, un sujet toujours d'actualité en raison des phénomènes de repli identitaire, des nouvelles formes de nationalismes, de fondamentalismes; mais également en raison de l'aspiration légitime de certains peuples à plus de démocratie et de justice sociale (guerres incessantes dans plusieurs régions du monde).

\section{L'objet " patrimoine martyr "}

Quand le patrimoine cristallise un sentiment d'appartenance (nationale, religieuse, idéologique), d'un groupe contre un autre groupe, quand on lui attribue une valeur symbolique, religieuse, identitaire (et/ou économique), il devient un objet de souveraineté, donc de convoitise. Ainsi peut surgir cette "haine monumentale » analysée dans le livre de François Chaslin, en référence aux destructions intentionnelles des villes de l'ex-Yougoslavie ${ }^{3}$. L'histoire du patrimoine architectural détruit violemment et peu à peu reconstruit, est comme une forme de fabrique continue. Fabrique qui est comme un tissage, où la trame est tradition et la chaîne les diverses formes de modernités et de ruptures.
J'associe l'idée du patrimoine martyr avec les sites qui ont été détruits en tant que monuments historiques, ou pour I'histoire récente en tant que patrimoine mondial (World Heritage, UNESCO). Même si une grande partie des destructions de la Seconde Guerre mondiale sont plutôt à définir comme dommages collatéraux, résultants des bombardements systématiques (depuis le sol ou aériens), bombardements qui ne les visaient pas de façon spécifique. II est utile d'identifier, au cas par cas, I'origine, la stratégie et la technique de la destruction durant la guerre (destruction intentionnelle, destruction «non-intentionnelle», dynamitage, bombardement aérien, pilonnage depuis le sol). Dans le cas de Cologne, de Saint-Lô ou de Rimini, villes martyrs presque entièrement rasées, les monuments historiques n'étaient «que I'un » des éléments à détruire pour miner l'ennemi et le contraindre à se rendre. Dans les cas de Dresde et de Coventry, la destruction des monuments historiques n'a pas été uniquement le résultat d'effets collatéraux, mais était bien une destruction intentionnelle; les frappes (ou tapis de bombes) mirant à la destruction symbolique d'une civilisation. II existe donc plusieurs façons de martyriser le patrimoine. Le dynamitage de monuments, via des explosifs posés sur l'édifice et commandés à distance, est bien une forme de destruction volontaire, et non pas un effet collatéral. C'est le cas pour des monuments comme l'église abbatiale de Lessay dans la Manche, ou les anciens ponts de Florence et de Vérone (ponte a Santa Trinità, ponte del Castelvecchio, ponte di Pietra).

Dans les guerres d'aujourd'hui (mais cela n'est pas nouveau), on ne se contente plus de tuer son voisin, de détruire ses équipements stratégiques (ports, industries,

3. François Chaslin, Une haine monumentale. Essai sur la destruction des villes en ex-Yougoslavie, Paris, Descartes \& Cie, 1997. La géopolitique de la haine monumentale continue, en témoigne notamment l'ouvrage récent de Jean-Pierre Payot, La guerre des ruines, archéologie et géopolitique, Paris, Choiseul, 2010. 
stocks, gares...), c'est aussi les symboles de l'identité culturelle d'un peuple qui sont visés. Comme le dit la chercheuse Dacia Viejo Rose ${ }^{4}$, "en période de conflit, les belligérants arment la culture. Ils l'utilisent telle une bombe à retardement dont ils ont réglé I'heure et le jour de l'explosion... Le patrimoine culturel n'est pas seulement matériel, il se compose d'une part d'intangible et de symbolique ». On pense à la destruction du pont de Mostar en 1993 ou à celle des grands bouddhas de Bamyan en 2001: deux destructions filmées et médiatisées en guise de propagande, dont l'objectif est de détruire "l'autre » dans ce qu'il a de plus précieux, sa mémoire, ses reliques, ses œuvres d'art.

Il faut donc clarifier les discours qui sont à l'origine d'actions comme s'approprier un monument historique, le détruire en partie ou complétement, le conserver (donc le restaurer), le transformer et le mettre en valeur. Mais la réalité est bien souvent plus complexe et hybride que le discours théorique ou doctrinaire. L'amour pour un monument ou la haine de ce même monument peut coexister au même moment, dans un même lieu. Ainsi, démolir ou mal restaurer un monument peut parfois participer des mêmes principes symboliques de domination d'un groupe sur un autre, d'une époque sur une autre époque, d'une idéologie sur une autre idéologie, par des phénomènes de réappropriation ${ }^{5}$. Par exemple, Dacia Viejo Rose écrit sur l'Espagne franquiste: "Une fois la guerre civile gagnée, Franco a instauré une dictature. Pendant des décennies, il a modelé le patrimoine culturel de l'Espagne selon sa vision, en niant celle des vaincus ${ }^{6}$. " Ce type phénomène a la vie dure dans beaucoup de pays: les Tibétains ou les
Ouïghours " en-chinoisés », les Kurdes ou les Arméniens «turkéifiés »..., des formes de domination agissant au détriment de la diversité culturelle, donc au détriment du patrimoine matériel et immatériel. Les monuments, comme les sites archéologiques, touchent par leur dimension identitaire et par leur présence physique très forte au contrôle symbolique d'un territoire. La " main mise " sur les valeurs symboliques (religieuses, nationales, ethniques) d'un territoire procède d'un ensemble de mécanismes sociologiques complexes, que j'ai nommé « le paradigme de Mostar ».

La question des valeurs attribuées au monument a été analysée en profondeur notamment par I'historien autrichien Aloïs Riegl, dans son célèbre essai Der moderne Denkmalkultus (Le Culte moderne des monuments, 1903), véritable épistémologie des notions de monument et de monument historique. Avec le passage du monument historique au patrimoine, cette question des valeurs se complexifie davantage, s'étend, et se relie notamment aux phénomènes d'appropriation. Certains concepts d'Aloïs Riegl n'ont pas toujours été correctement traduits en français, ce qui peut prêter à confusion. Notamment le Gewolltes Denkmal traduit comme «monument intentionnel » et le Gewordenes Denkmal traduit comme « monument non intentionnel »; alors qu'en réalité cela se traduit «monument voulu » et «monument devenu »; ce qui est assez différent ${ }^{7}$.

Comme l'explique Gabi Dolff-Bonekämper ${ }^{8}$, le patrimoine architectural comprend diverses dimensions comme: le lieu (genius loci), la forme de l'objet (art, histoire des formes, styles, etc.), son statut (une église, un château, un pont, etc.), la sémantique (signifié, signe,
4. Dacia Viejo Rose, Reconstructing Spain: Cultural Heritage and Memory after Civil War, (Sussex Academic Press 2011), PhD du McDonald Institute for Archaeological Research, université de Cambridge; elle travaille depuis quatre ans sur un projet interdisciplinaire nommé CRIC, "Cultural Heritage and the Reconstruction of Identities after Conflict ».

5. Phénomène très ancien, dans l'Égypte pharaonique, les monuments étaient modifiés (gommage des cartouches...) au profit du nouveau pharaon.

6. Dans Fabrice Praz, «La culture en période de guerre: une bombe à retardement ", Rue89, Genève, 15 avril 2011.

7. Point à propos de Aloïs Reigl vu lors d'un entretien avec Gabi y-Dolff-Bonekämper (conservatrice des monuments historiques et professeure à l'université de Berlin), que je remercie pour ses précieux conseils.
8. Adrian von Buttlar, (dir.), " La perte du Monument comme une construction sociale », (p. 134-165), texte en allemand de G. Dolff-Bonekämper, Monuments lieux de reconstruction du culte factice de monuments architecturaux. Une anthologie, Gütersloh, Berlin, Bâle, Birkhäuser, 2010.

Nicolas Bullock et Luc Verpoest, (dir.), Living with History, 1914-1964, Leuven University Press, Leuven, 2011 (issu du Symposium de Louvain, contribution, Histoire sans abri, de G. Dolff-Bonekämper. 
marque), les acteurs qui l'ont pensé et bâti (commanditaires, artistes parfois mythiques ou moins connus, artisans, etc.), et enfin son nom. Ces diverses réalités imbriquées, sont présentes dans ce que j'ai nommé le «patrimoine objet», le "patrimoine sujet», le « patrimoine otage » le « patrimoine martyr ».

\section{Le " patrimoine objet "}

Il s'agit de la consistance matérielle d'un monument historique formée parl'ensemble de ses systèmes constructifs depuis ses fondations jusqu'à sa couverture en passant par ses murs, colonnes, voûtes, planchers, charpentes, décors, enduits, fenêtres, corniches, etc. Sur le patrimoine objet, les spécialistes chargés de son entretien peuvent se comprendre, peuvent dialoguer car ils sont dans une démarche pluridisciplinaire à caractère scientifique. Ainsi, l'archéologue, l'architecte des monuments historiques, I'historien de l'art, le chimiste, le spécialiste des matériaux, le restaurateur de peintures murales, peuvent dialoguer et se comprendre (même si ce n'est pas toujours le cas); être complémentaires pour la question de l'entretien et de la restauration. Le patrimoine objet comprend le lieu, la forme et le statut. On restaure le patrimoine objet pour répondre à des questions architecturales, techniques, artistiques et fonctionnelles ${ }^{9}$. En résumé le patrimoine objet, c'est l'architecture et sa construction.

\section{Le " patrimoine sujet "}

Il s'agit de la manière dont une société va s'adresser à son patrimoine, à ses monuments, comme à un sujet porteur d'un ensemble complexe de valeur, de symbole, de croyance. C'est l'éclairage subjectif qu'une société va

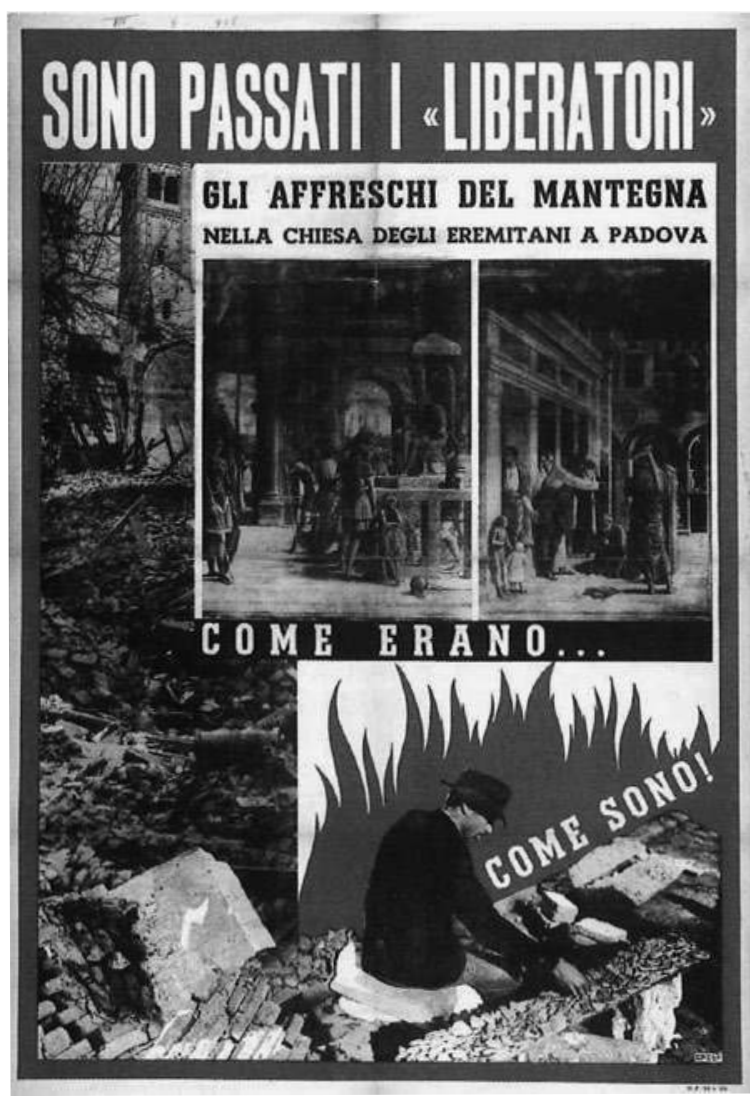

Padoue, église degli Eremitani, cappella Ovetari, affiche dénonciatrice des alliés qui ont bombardé l'église et les fresques d'Andrea Mantegna (traumatisme, perte chaude).

Sources: Padoue, Museo del Risorgimiento.

9. Raisons multiples comme: usure du temps, mauvaises réparations, manque d'entretien, non adaptation à une fonction nouvelle, adaptation aux normes sans cesse en évolution (énergétiques, sismiques, accessibilité des personnes à mobilité réduite, incendies, etc.). 
projeter sur son patrimoine. C'est également la reconnaissance individuelle et/ou collective de tel monument en vue d'une appropriation ou d'une "évaluation critique » (évaluation $\rightarrow$ valeurs). Dans ces catégories de valeurs, on trouve notamment: la valeur d'histoire, la valeur d'art, la valeur nationale ou religieuse, la valeur locale et ou ethnique, la valeur économique, la valeur de nouveauté, la valeur d'ancienneté, la valeur stylistique... Le patrimoine sujet implique de restaurer, de modifier (ou de démolir) un bâtiment (ou un site) pour répondre à des questions d'interprétation (ou de jugement) sur la question des valeurs.

Le patrimoine-sujet est traditionnellement étudié par les architectes, les archéologues et les historiens de l'art. Mais particulièrement depuis les années 1980, il est entré dans le domaine des sciences humaines et sociales ${ }^{10}$, devenant ainsi un sujet d'étude pour les chercheurs en géographie, en histoire, en anthropologie urbaine, en sociologie. La volonté de "marquage » de tel groupe social ou de tel pouvoir va aussi avoir des effets sur un patrimoine ${ }^{11}$. Enfin, l'évolution dans I'historiographie (valorisation/dévalorisation d'une époque par rapport à une autre) ou dans la recherche archéologique, peuvent avoir des effets positifs ou négatifs, voir destructeurs sur tel ou tel patrimoine. Le patrimoine sujet comprend la sémantique, les acteurs et le nom. En somme le patrimoine sujet, c'est l'ensemble des discours, des valeurs et des idéologies projetées sur un monument historique.

\section{Le " patrimoine otage"}

II s'agit d'un monument ou d'un site historique pris dans des phénomènes sociétaux qui le mettent en danger, avec un risque de dégradation (voir de destruction) à plus ou moins long terme. Parmi ces phénomènes sociétaux, il n'y a pas que la guerre, il y a aussi le tourisme de masse. L'inscription d'un site ou d'un monument sur des listes de protection (listes nationales ou internationales) n'est malheureusement pas toujours un outil permettant d'assurer sa conservation. La destruction du patrimoine otage peut-être volontaire, comme dans le cas des guerres dites «civiles». Elle peut également être un mélange de volontaire et d'involontaire, comme dans le cas d'effets collatéraux non contrôlés d'une guerre. La puissance financière, comme durant la période des «Trente Glorieuses » en Europe, peut-être destructrice du patrimoine; comme par exemple, le centre ancien de Bruxelles en bonne partie détruit par la spéculation urbaine à partir des années 1950. Les idéologies sont aussi des moteurs de destructions. Notamment la doctrine des CIAM, exprimée dans la « charte d'Athènes » (1933, publiée en 1941) est le support théorique de plans d'urbanisme prévoyant la destruction de centres historiques au profit d'un aménagement " tout moderne », avec la pénétration de voies rapides dans des centres historiques, la séparation des flux, l'isolement de monuments historiques au milieu de nouveaux bâtiments hors d'échelle, etc.

À ce sujet, l'exemple de la reconstruction urbaine de Cologne est significatif. Là, comme dans beaucoup de villes après 1945, la doctrine inspirée par les CIAM a parachevé le travail de destruction bien entamé par les bombardements de la Seconde Guerre mondiale. Le régime communiste de l'ex-URSS a pu également décider, au cas par cas, de transformer radicalement le territoire en détruisant au passage nombres de monuments (églises, monastères) et de quartiers anciens. En résumé, le
10. Depuis La notion de patrimoine, (André Chastel et Jean-Pierre Babelon, 1980), et les Lieux de mémoire, (Pierre Nora, 1997), L'allégorie du patrimoine, (Françoise Choay, 1992), un vaste champ de recherche s'est développé autour du patrimoine comme sujet d'études en sciences humaines. David Lowenthal, The Heritage Crusade and the Spoils of History (D. Lowenthal, 1998),

François Hartog, Régimes d'historicité, (F. Hartog, 2003), et les travaux de Lucie K. Morisset, Maria Gravari-Barbas,
Vincent Veschambre, Jean-Yves Andrieux, Dominique Poulot, Simone Ricca, David Berliner.

11. Voir à ce sujet l'ouvrage de Vincent Veschambre, Traces et mémoires urbaines. Enjeux sociaux de la patrimonialisation et de la démolition, Rennes, Presses universitaires de Rennes, 2008. 


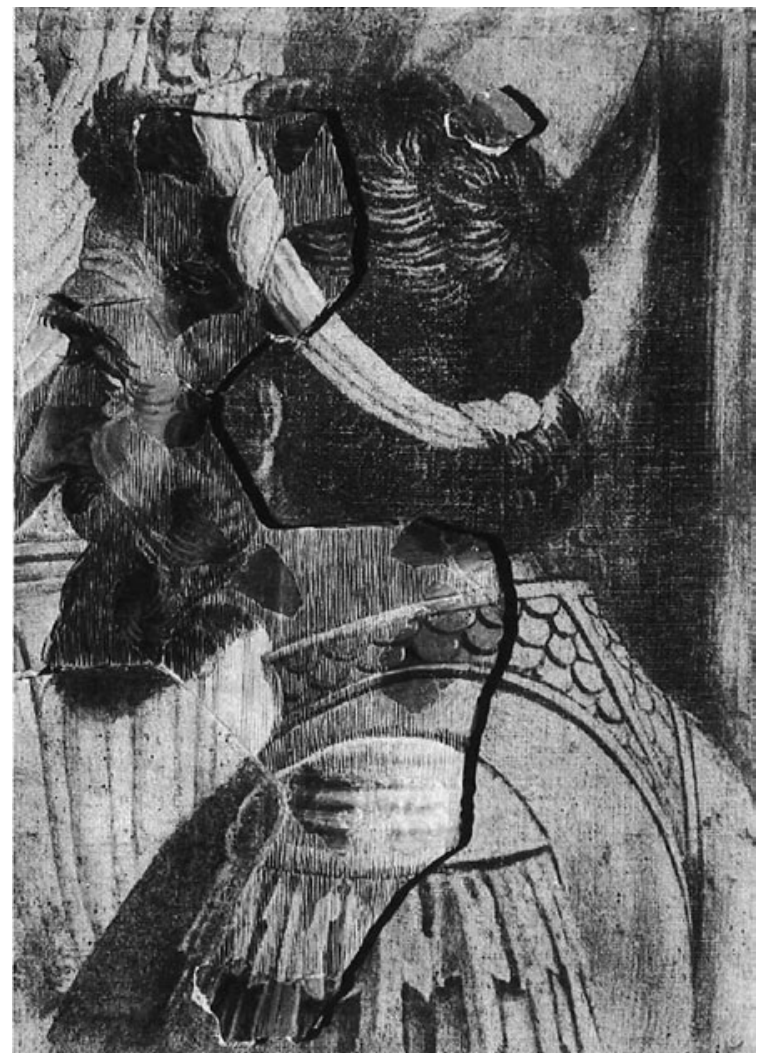

Padoue, église degli Eremitani, cappella Ovetari, fresques d'Andrea Mantegna, Il Centurione (détail) après les premières restaurations par réintégration des lacunes et techniques du " tratteggio " (Cesare Brandi).

Sources archives: MIBACT-SBAP-VeBPT-AF, Archivio Fotografico. patrimoine otage, c'est la ville ancienne (celle d'avant 1940) prise dans l'étau de la pression économique et politique du présent. II faut souligner le fait que le patrimoine sujet et le patrimoine otage sont comme incarnés dans le patrimoine objet. Celui qui étudie le patrimoine objet (donc l'architecture) doit être conscient de ses diverses dimensions (lieu, forme, statut, sémantique, acteurs, nom), à l'instar de "l'exégèse critique » qui étudie les textes anciens, et notamment les textes bibliques.

\section{Le " patrimoine martyr "}

Dans son sens originel, martyr vient du grec martur, forme tardive de martus, marturos «témoin». Pour les chrétiens c'est celui qui témoigne de la vérité et de la foi par son sacrifice.

"Le martyr est une sublimation, une sublimation corrosive. C'est une torture qui sacre » écrit Victor Hugo (Les Misérables, V, VI, IV). Le terme est aussi lié à l'idée de "se souvenir ». La plupart des saints des premiers siècles du christianisme sont des martyrs. Le culte des martyrs, la martyrologie, est lié au culte des reliques. Pour le croyant, la relique permet à l'homme mortel de se relier à ce que Karlfried Graf Dürckheim nomme "le tout autre ». La relique (les ossements de saint Nicolas, la robe de bure de saint François d'Assise...) est censée aider le croyant à se souvenir de la vie exemplaire et spirituelle du saint, et de là, à la dimension immortelle de Jésus-Christ, qui passe par son sacrifice et son martyr. Ainsi, les ruines (de guerre, comme les ruines archéologiques) sont une trace qui aide l'homme à entrer dans la profondeur du temps, dans « le tout autre » (dimension culturelle, esthétique et spirituelle). 
66 Les Cahiers de la recherche architecturale et urbaine - n०30/31 $\quad$ Trajectoires doctorales 2

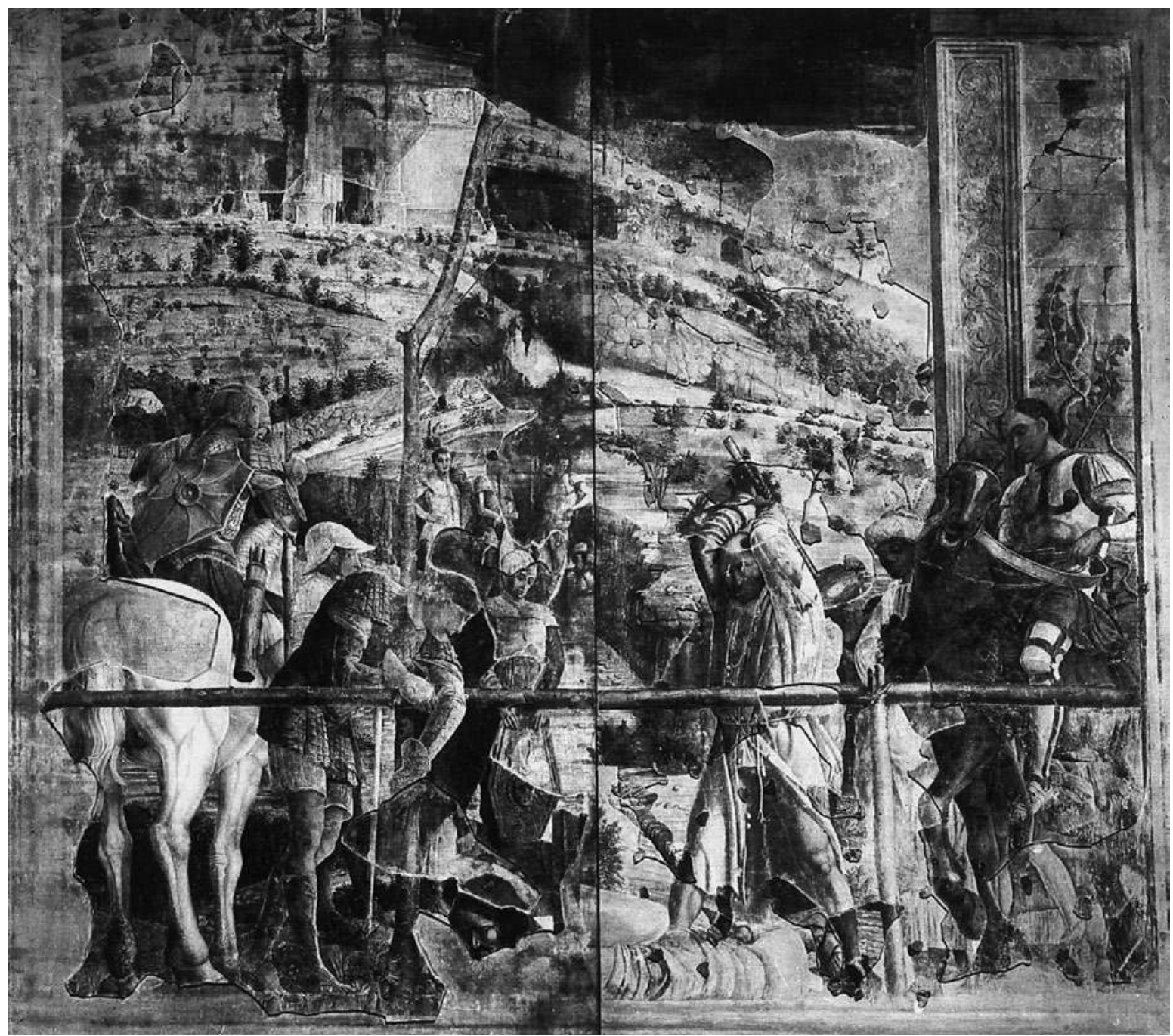

Padoue, Cappella

Ovetari, fresques

d'Andrea Mantegna,

un panneau après

a restauration par

réintégration des

lacunes (Restauration

ICR, Cesare Brandi).

Sources archives:

MIBACT-SBAP-VeBPT-AF,

Achivio Fotografico. 
Padoue, degli Eremitani, aujourd'hui Museo Civico di Padova. Vue du grand cloître reconstruit (architectes : Franco Albini et Franca Helg). Photo N. Detry, 2013.

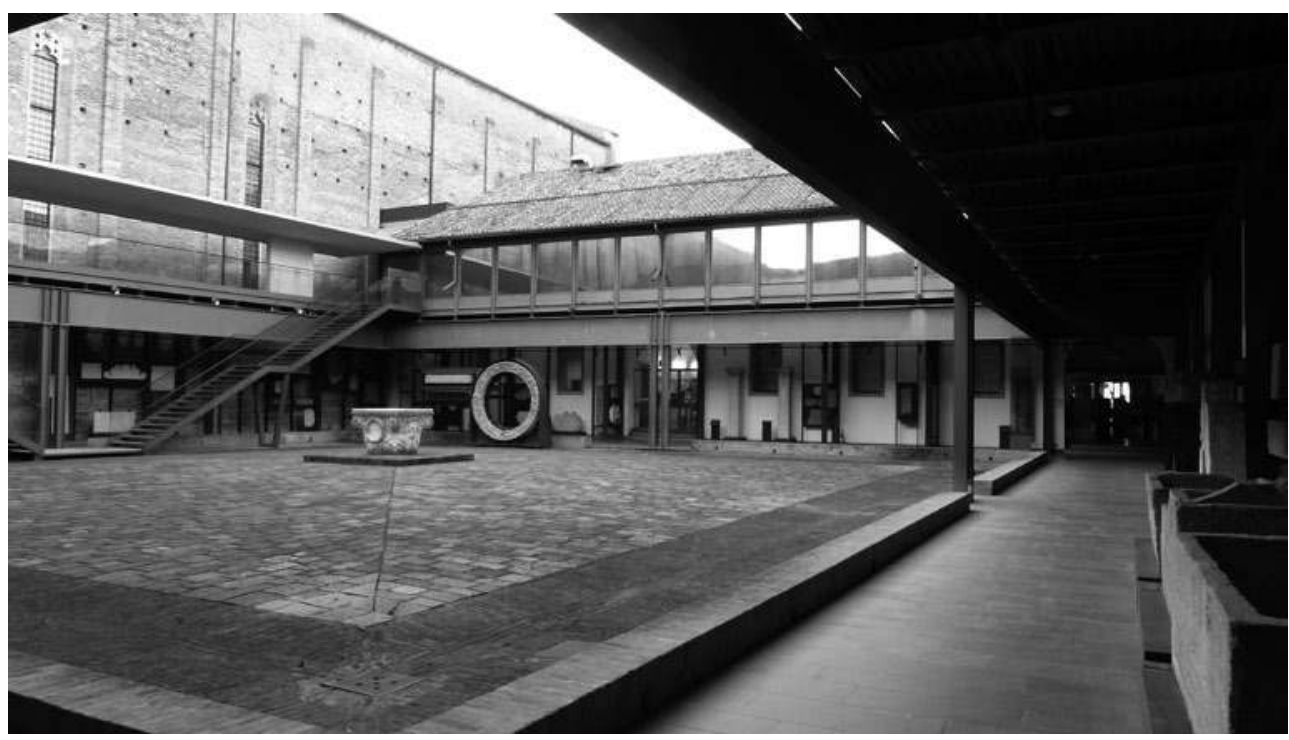

Le terme patrimoine martyr est donc utilisé à dessein, dans ces deux acceptations, celle de "torturé à mort " et celle de "témoin", qui est la trace d'une mémoire à transmettre. II s'agit ainsi de l'étape ultérieure du patrimoine otage, quand la destruction (partielle ou totale) est effective et est due à un conflit armé. Je prends comme point de départ le patrimoine martyr, afin d'analyser ce qui se joue après: la restauration, forme de résurrection. Si le patrimoine martyr est un fait sociétal très ancien, il est malheureusement encore très actuel. Les exemples récents sont innombrables (Sarajevo, Mostar, bouddhas de Bamiyan) et actuel (la Syrie, le Mali, I'Irak, l'Égpte, I’Ukraine?).

\section{La problématique}

La restauration des monuments historiques en Europe à l'issue de la Seconde Guerre mondiale

La problématique centrale de cette recherche repose sur ce qui va se passer après la destruction, quand celle-ci est due à une guerre ou à une forme de conflits armés. Faudra-t-il, après ce traumatisme, conserver l'édifice ruiné, le reconstruire, le restaurer, le consolider? Si la restauration est légitime, quelle forme va-t-elle prendre? Où, pourquoi, comment, avec quelles méthodes, quelles théories, quel discours, quels moyens, quels acteurs? Je postule que ce qui nous guide en matière de restauration, n'est pas une doctrine, mais plutôt un 


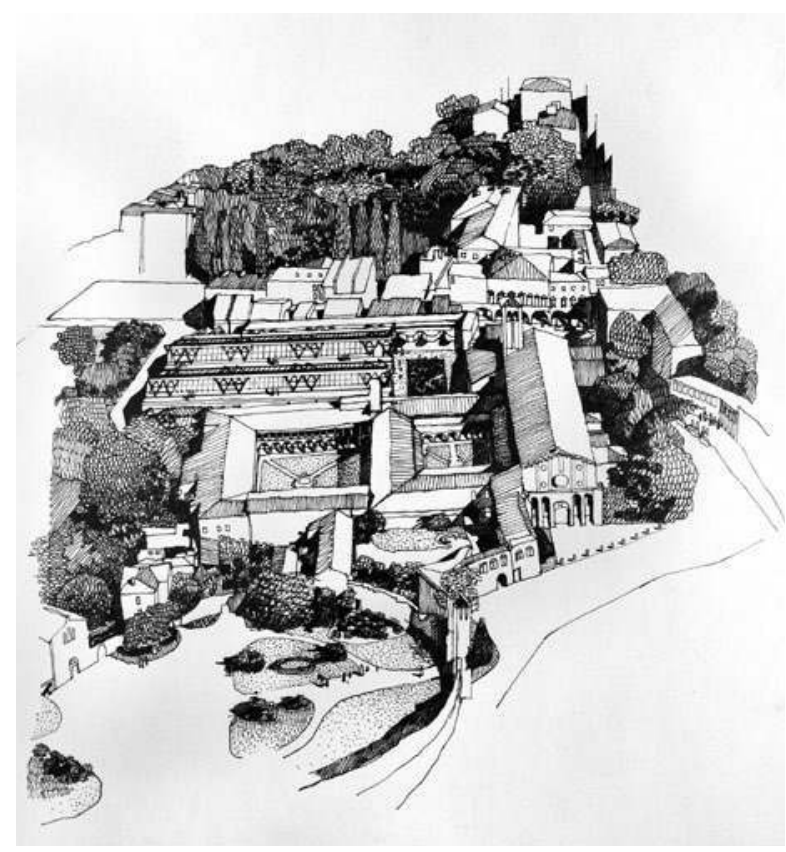

ensemble de «lignes guides », des méthodes de travail qui sont propres à notre discipline. Des méthodes ont été théorisées (à un très haut niveau d'élaboration), justement en réponse aux destructions de la Seconde Guerre mondiale, en particulier en Italie. Nous verrons de quelle façon cette approche a été nouvelle par rapport aux expériences de la première moitié $d u x^{e}$ siècle, expériences rassemblées notamment en 1931 dans la "Charte d'Athènes pour la restauration des monuments historiques ${ }^{12}$ » et nommées en Italie « restauro filologico et restauro scientifico ».
Padoue, Eremitani, coupe axonométrique, projet architecte M. Sacripanti, lauréat du concours de 1967. Sources: Archivio Generale del Comune di Padova, fondo lavori pubblici.
Padoue, Eremitani, croquis d'ensemble: à droite, l'église, au centre les 2 cloîtres, à gauche en bas, la Cappella degli Scrovegni, architecte Maurizio Sacripanti, lauréat du concours de 1967. Au fond de l'image, projet d'extension du musée. F. Albini avait également conçu cette extension (projet publié dans plusieurs revues d'architecture dont Casabella). 47 ans après une version réduite de cette extension est finalement mise en chantier
(2013-2014) selon un projet qui s'inspire du dessin de F. Albini (" perte retrouvée "), à la demande du Sovritendente Dott. Arch.

Antonella Ranaldi. Sources: Archivio Generale del Comune di Padova, fondo lavori pubblici.

\section{L'intervention sur les monuments historiques} détruits pendant la guerre est particulièrement révélatrice (selon des temporalités variées) des débats et des controverses qui concernent la restauration en général. Ces débats sont encore d'actualité aujourd'hui, exactement cinquante ans après le célèbre « $2^{\mathrm{e}}$ Congrès international des architectes et techniciens des monuments historiques ". C'est là, à Venise en 1964, que les responsables venus du monde entier ont ressenti la nécessité morale et spirituelle de confronter les méthodes et les pratiques; ils étaient encore

12. Les acteurs de la Charte $d^{\prime}$ Athènes des monuments historiques de 1931, sont essentiellement français et italiens (parmi eux Leopoldo Torres Balbás, Paul Léon et Gustavo Giovannoni). 


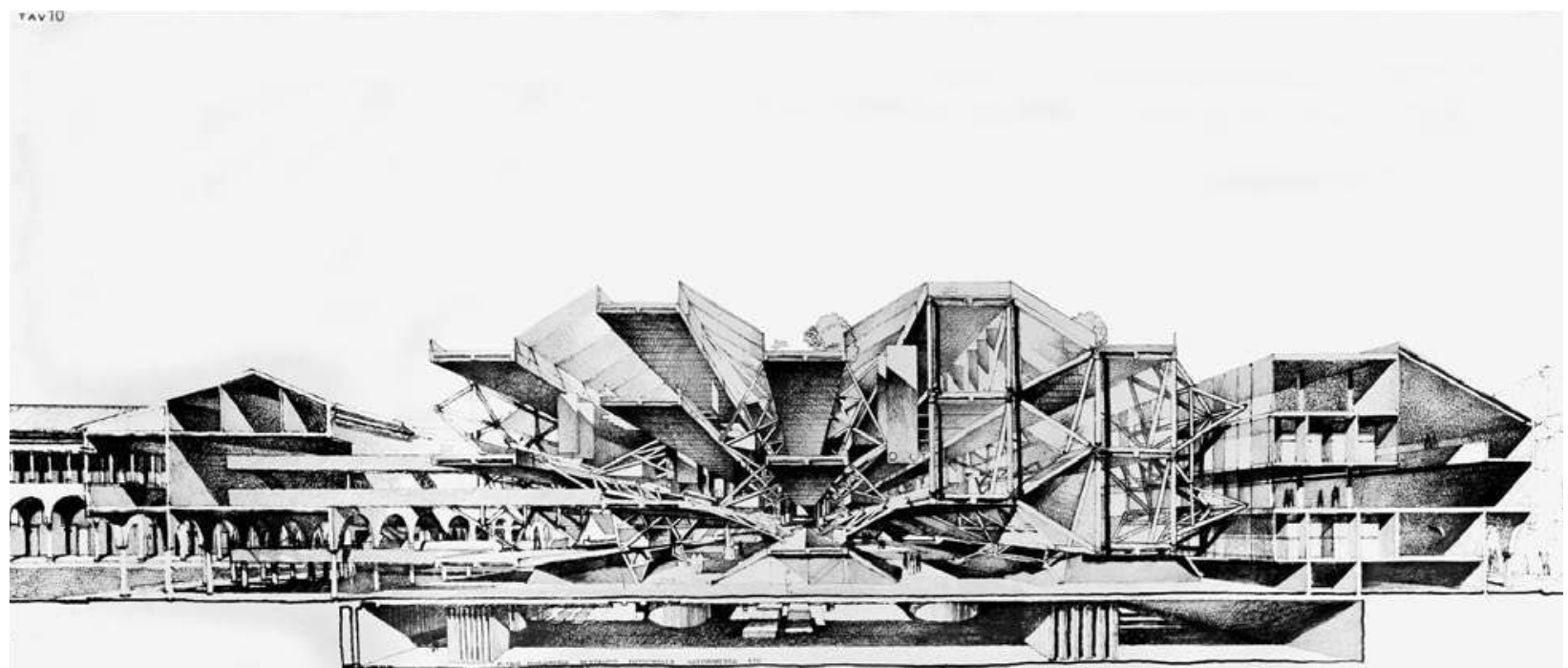

en prise avec la reconstruction du patrimoine européen, chaque pays selon ses propres temporalités.

Ces fondements théoriques doivent donc être retrouvés dans un corpus de textes et d'exemples concrets situés dans la période de la Reconstruction post-bellico, soit essentiellement de 1945 à 1972 (année de la Convention du Patrimoine mondial de L'UNESCO) ; période qui comprend le moment clé de mai 1964 (charte de Venise). Après la guerre et compte tenu de I'ampleur des destructions, la nécessité s'est imposée d'intenses échanges internationaux pour tenter de construire de façon concertée de nouvelles méthodes et techniques pour la restauration monumentale (création de I'Icomos et de I'Iccrom). Nous faisons notamment I'hypothèse que "la restauration critique " (il restauro critico) approche issue de quelques acteurs italiens ${ }^{13}$ (non majoritaires) prend une place particulière dans ces débats; même si chaque pays tend à conserver une certaine identité culturelle, une forme d'indépendance en matière de restauration. Nous faisons également I'hypothèse que l'œuvre de l'architecte turinois Andrea Bruno $^{14}$ permet de lire ce qui se joue durant cette
13. Le principal théoricien du restauro critico en architecture est Renato Bonelli (1911-2004). Voir à ce sujet Pierre Prunet et Nicolas Detry, Architecture et restauration, Paris, Éditions de la Passion, 2000. Roberto Pane (1897-1987) a également joué un rôle majeur dans ce domaine. Aujourd'hui, le professeur architecte Giovanni Carbonara développe cette approche sensible avec une attention majeure à la conservation, ainsi qu'une relation critique et créative avec notre monde contemporain.
14. Sur l'architecte Andrea Bruno, voir notamment le livre de Mario Mastropietro (dir.), Oltre il restauro. Architettura tra conservazione e riuso. Progetti e realizzazioni di Andrea Bruno (1960-1995), Milan, Lybra Immagine, 1996; ou I'article de Francesca Brancaccio et Nicolas Detry, "Andrea Bruno, l'observatoire de l'architecture », $A+, n^{\circ} 165$, Bruxelles, 2000, p. 64-93. 


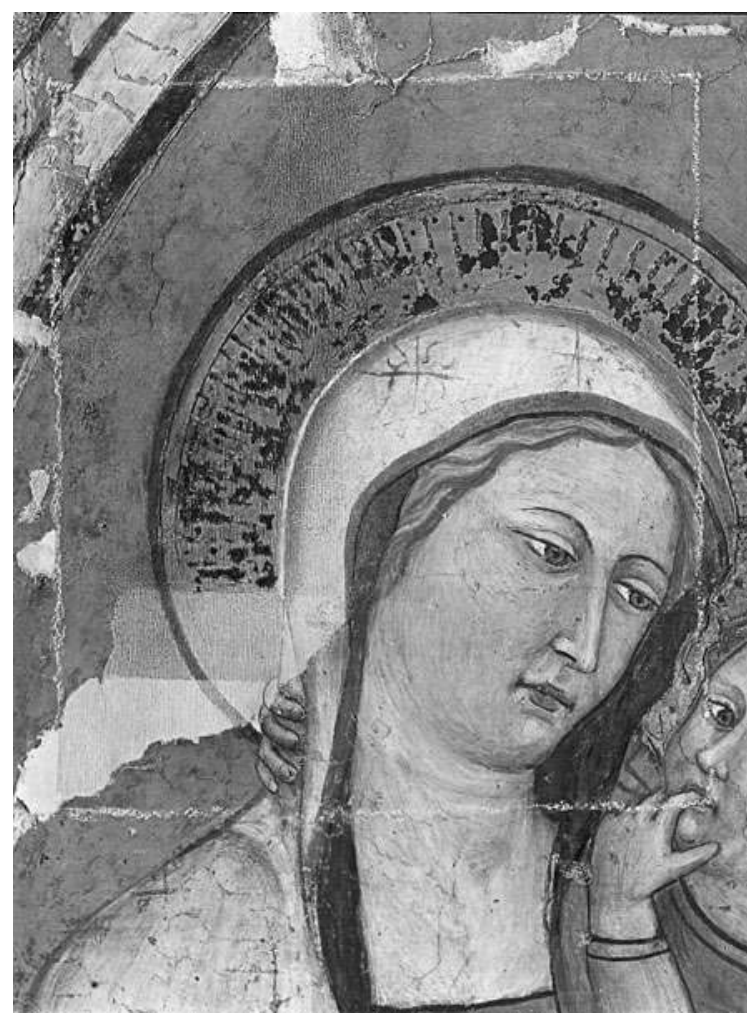

Église de Tuscania (Italie), Vierge à I'enfant: exemple de réintégration de lacunes avec la technique du tratteggio, en cours d'exécution. période, cela jusqu'à aujourd'hui, à un niveau international. Par-delà les intentions, les affichages, les affiliations à des théories, nous chercherons à montrer la pratique de la restauration post-bellico et sa relative convergence (similitudes) dans les divers contextes culturels choisis (Allemagne, France, Italie). Cela se vérifie, même s'il faut y apporter une infinité de nuances, durant les «Trente Glorieuses », que nous allons nommer par convention la "période chaude ». Si les Italiens ont joués un rôle de premier plan (par exemple au moment de la charte de Venise), c'est probablement parce que l'Italie a toujours eu une forme de primauté mondiale dans le domaine de la restauration des biens culturels en général. Les seize articles de la charte sont une forme de brillante synthèse, fruit de l'expérience de grands acteurs italiens et internationaux ${ }^{15}$.

\section{Sur l'actualité de ces questions}

Aujourd'hui encore, pour diverses raisons, les conséquences de la Seconde Guerre mondiale sur les monuments historiques sont des blessures non encore entièrement cicatrisées. Des questions restent ouvertes, des projets sont en marche, des sujets "chauds» sont débattus. Si cela est moins vrai dans un pays centralisé comme la France, où le service des Monuments historiques a réalisé efficacement l'essentiel des reconstructions entre 1945 et 1972, il est frappant de constater qu'ailleurs, par exemple en Allemagne, en Italie ou dans les pays de l'ex-URSS, des chantiers de reconstruction post-bellico viennent juste d'être achevés, sont en cours de réalisation et sont encore à venir. À ce propos, les experts allemands parlent même

15. Pour l'architecture, on peut citer quelques acteurs de premier plan: Pietro Gazzola, Alfredo Barbacci, Renato Bonelli, Roberto Pane, Giuseppe Zander, Liliana Grassi, etc. Pour la conservation des œuvres d'art et des peintures murales, l'œuvre de Cesare Brandi est fondamentale ainsi que le rôle de Paul Philippot. L'ensemble des contributions ayant été synthétisé par Raymond Lemaire. 
d'une "nouvelle vague de reconstruction ${ }^{16}$ " depuis 1990, comme en témoignent la reconstruction actuelle du château de Berlin, ainsi que les reconstructions en cours de Potsdam.

En ex-Yougoslavie, pour ne citer que l'exemple de Sarajevo, la bibliothèque (et hôtel de ville) est en cours de reconstruction depuis 2010. Cette restauration du Sarajevska Vijcenica, bombardé le 26 août 1992, est officiellement une reconstruction à l'identique dont le chantier sera achevé en 2014, au moment des célébrations du centenaire de l'assassinat à Sarajevo de l'archiduc François-Ferdinand, événement considéré comme déclencheur de la Première Guerre mondiale.

\section{Évocations des méthodes et des sources}

Le principe adopté est le suivant: pour comprendre I'histoire de la restauration post-bellico, il est nécessaire de voir ce qui s'est passé dans au moins trois grands pays: I'Allemagne, la France et I'Italie. Parce que ces trois pays ont un vaste corpus d'expérience et de recherche (universitaires et professionnelles), dans la discipline qui nous occupe, tout en conservant chacun une spécificité culturelle du fait de l'histoire politique propre à chaque nation et à chaque région. La spécificité régionale étant, on le sait, très forte en Allemagne et en Italie et moins forte en France ${ }^{17}$.

Les cas d'études ont été volontairement choisis dans le domaine des édifices classés comme monuments historiques, selon deux typologies: I'architecture sacrée (églises, monastères) et les bâtiments civils publics (musée, bibliothèque, hôtel de ville). Pourquoi choisir uniquement deux types de bâtiments, alors que la guerre a tout détruit

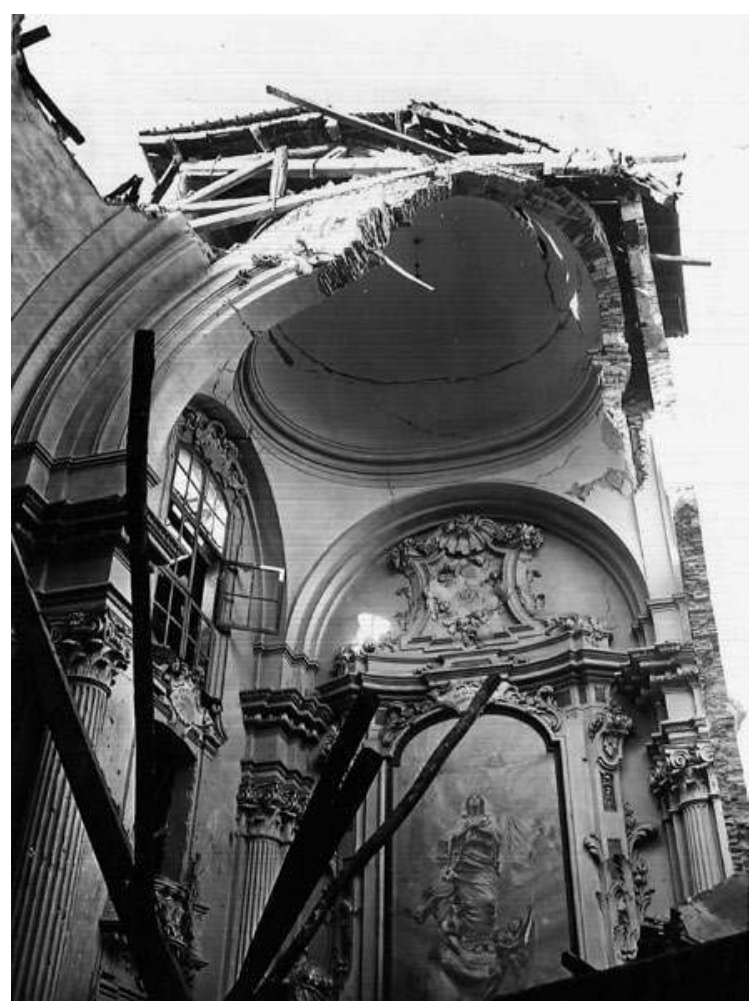

\begin{tabular}{|c|c|}
\hline $\begin{array}{l}\text { Bologne, oratoire } \\
\text { de } S \text {. Filippo Neri, } \\
\text { état en } 1944 \text { après } \\
\text { les bombarde- }\end{array}$ & $\begin{array}{l}\text { les voutes } \\
\text { et une portion } \\
\text { de la coupole } \\
\text { de l'abside. } \\
\text { Sources: } \\
\text { MIBACT-SBAP, } \\
\text { Bo. AF, }\end{array}$ \\
\hline
\end{tabular}

16. Winfried Nerdinger, Markus Eisen et Hilde Strobl (dir.), Geschichte der Rekonstruktion. Konstruktion der Geschichte, Munich, Prestel, 2010, catalogue d'exposition du Centre d'architecture de Munich: I'auteur a fait traduire par Maricke Hofmann une série d'articles de ce livre très important.
17. D'autres chercheurs, comme Andrea Pane, étudient cette question pour l'Angleterre qui a également une expérience de reconstruction post-bellico très importante. Quelques cas et acteurs anglais seront mobilisés pour éclairer notre analyse. 
Bologne, oratoire de S. Filippo Neri, la nef après la restauration conçue par l'arch. Pier-Luigi Cervellati. On note que P. L. Cervellati a conservé la trace du premier chantier de restauration mené par Alfredo Barbacci durant la " période Chaude" (murs de briques et colonnes inachvées en béton)

Sources: P-L Cervellati, L'ex Oratorio di San Filippo

Neri restituito alla città, Bologne, Costa, 2000.

Photo Diego Tabanelli (SBAP-Bo.)

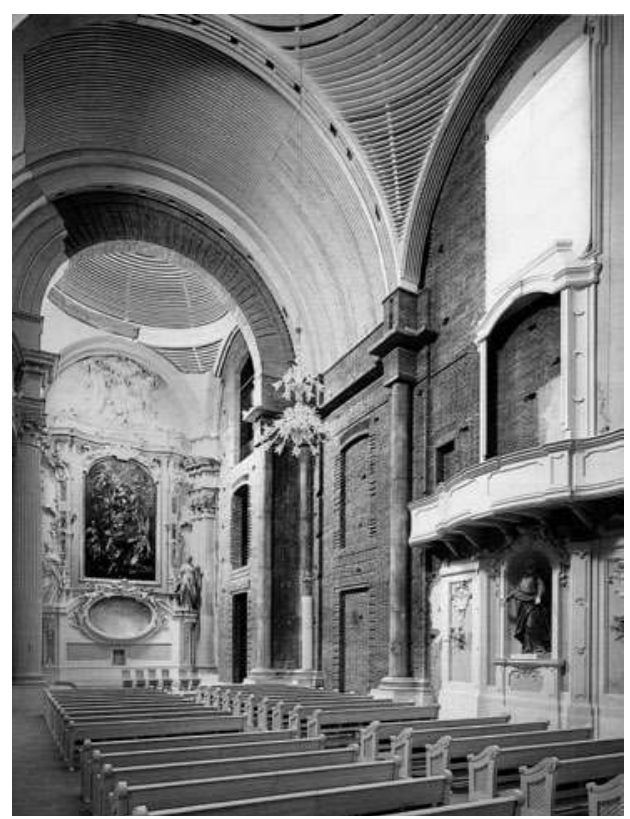

Bologne, oratoire de S. Filippo Neri, détail des voûtes, avec la réintégration architecturale des lacunes, comparable aux réintégrations des peintures murales. Sources: P-L Cervellati, L'ex Oratorio di San Filippo. Photo Diego Tabanelli (SBAP-Bo.)

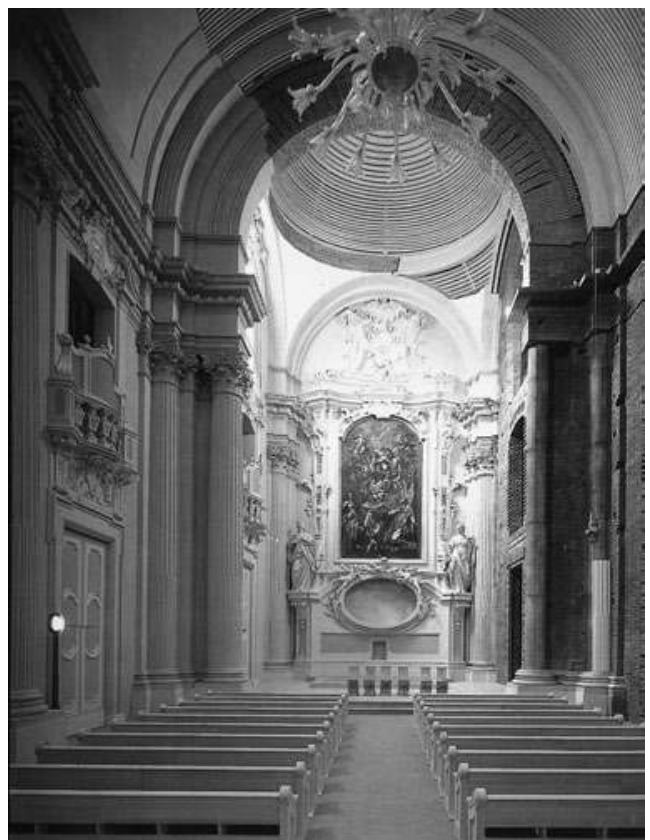

Bologne, oratoire de S. Filippo Neri, en 2000 , après la restauration conçue par l'architecte Pier-Luigi Cervellati (nouvel usage comme salle de spectacle et lieux de culture, maître d'ouvrage, Fondazione del Monte).

Sources: P-L Cervellati, L'ex Oratorio di San Filippo Neri restituito alla città, Bologne, Costa, 2000.

Photo Diego Tabanelli (SBAP-Bo.)

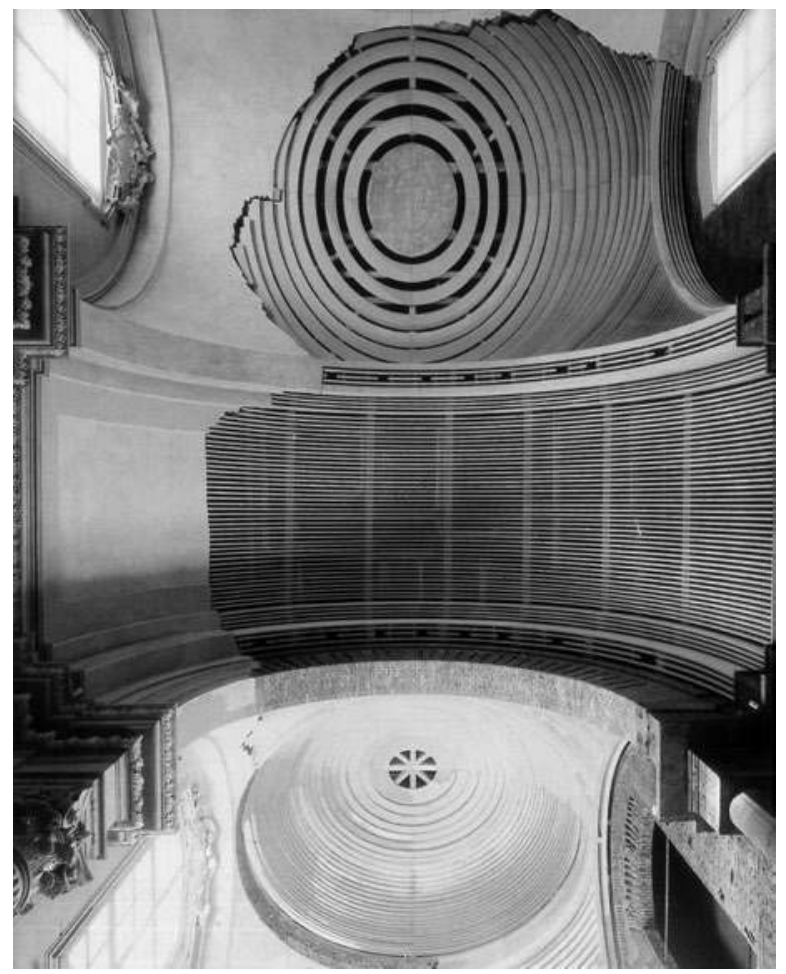


(les ponts, I'habitat, les gares, les usines, etc.)? Parce que j'entends apporter une contribution sur un sujet certes vaste, mais ciblé, lié à l'histoire de la restauration des monuments historiques. Parce que l'histoire de la reconstruction des villes, des tissus urbains de l'habitat (aussi des ponts) a été assez bien étudiée (notemment pars Danièle Voldman et Rémi Baudoui) ${ }^{18}$. Parce que les expériences des trois pays choisis restent encore trop étanches, circulent mal. Est-ce dû à la barrière des langues ou à la très forte identité et tradition de ces trois grands pays européens? Enfin, travailler sur deux types de bâtiments permet de comparer des choses comparables et de circonscrire le sujet. En outre, j'ai personnellement acquis, depuis 1994, une expérience professionnelle sur ce type de travaux, comme architecte spécialiste en restauration des sites et des monuments historiques; ayant étudié et travaillé en France, en Italie et en Belgique.

\section{Choix des études de cas et méthodes d'analyse des cas}

J'ai choisi de travailler sur deux à trois cas principaux par pays, avec d'autres cas évoqués pour renforcer l'analyse. Nous étudions notamment les cas suivants:

Allemagne : Cologne, l'église de Sankt-MariaIm-Kapitol, I'ancien monastère de Sankt-Columba (aujourd'hui musée Kolumba), I'église de Sankt-Alban au Gürzenich (l'ancienne laissée en ruine, la nouvelle bâtie avec les briques du théâtre bombardé de Cologne); Düren, l'église Sankt-Anna (architecte: Rudolf Schwarz); Munich, la Alte Pinakothek, l'église de Sankt-Bonifaz, la Glyptothèque, le palais de la Residenz; Berlin, le Neues Museum et l'église du Souvenir.
France: I'église de Saint-Malo de Valognes, l'église de Saint-Étienne à Strasbourg, l'église abbatiale de Lessay, I'église Notre-Dame de Saint-Lô, le clocher de l'église de Chantrigné, la salle des procurateurs du palais de Justice de Rouen.

Italie: à Padoue, l'église degli Eremitani avec la Cappella Ovetari et le Museo Civico; Rimini, le temple de Malatesta, le théâtre Galli avec les travaux en cours; Bologne, l'église et monastère de San-Francesco, le théâtre anatomique de I'Archiginnasio, I'Oratoire dei Filippini; Viterbo, la Cappella Mazzatosta.

Chaque cas est étudié selon différents critères complémentaires: historique, artistique et tecnique. Les liens entre la restauration des œuvres d'art (peintures murales en particulier) et la restauration architecturale sont analysés. La technique de construction d'un monument est importante car elle influe à la fois sur la façon dont il est endommagé, et sur la manière de le restaurer (possibilité de restaurer ou pas telle ou telle architecture, ayant telle technologie constructive). Une église en bois ou l'ossature métallique d'un pont risque de disparaître sous les flammes. Un bâtiment en brique cuite (ou crue) pourra mieux résister au feu ou à un bombardement. Les pierres d'un monument à stéréotomie de pierre peuvent être retrouvées autour de l'ouvrage dynamité, afin de tenter sa recomposition par anastylose. Les valeurs du monument (patrimoine sujet, nom, acteurs, significations) sont aussi prégnantes car elles influent sur l'approche de sa reconstruction, idem pour les pratiques sociales, l'usage, l'engagement citoyen, etc. Le type de destruction sera aussi étudié, sachant que les bombardements aériens n'ont pas les

18. Danièle Volman, La Reconstruction des villes françaises de 1940 à 1954, histoire d'une politique, Paris L'Harmattan, 1997 ; Dominique Borjot, Rémie Baudoui, Danièle Voldman (Dir.), La Reconstruction en Europe, 1945-1949, Bruxelles, Complexe; 1997. 
mêmes effets que les attaques depuis le sol (chars, obus, mitraillettes) et que le dynamitage d'un monument crée aussi des dégâts spécifiques.

\section{Diverses expériences et « températures " de la perte}

Enfin, les études de cas sont analysées selon différentes temporalités, en lien avec la notion de «perte». Au début, on peut parler de restaurations face à «une perte chaude " (réalisées dans une "période chaude " entre 1945 et 1972), par la génération qui avait connu ces monuments intègres avant la guerre. L'intense activité de reconstruction est comme alimentée directement par cette expérience de la perte, un traumatisme, avec pour les uns un sentiment très lourd de culpabilité. La dimension psychologique du drame de la perte constitue un substrat qui alimente le besoin de reconstruction. Après cette période, entre 1972 et 1990, des chantiers de reconstruction post-bellico se poursuivre poursuivent (notamment en Allemagne et en Italie). Si de façon schématique on peut parler de "période froide », (fin de la Guerre froide) on ne peut pas vraiment parler d'une " perte froide ». Car la notion de perte (d'un être cher comme d'un monument historique) n'est jamais froide, elle peut-être éventuellement "tiède » (enfouie dans le subconscient), mais rarement froide. Pour le $x x^{e}$ siècle, la "période chaude » devrait être en réalité celle qui va de 1914 à $1972^{19}$. Mais j'ai choisi de travailler essentiellement sur ce qui se joue après 1945.

J'ai nommé la période qui va de 1990 à aujourd'hui, " la nouvelle période chaude ». Car elle correspond à une période de restauration post-bellico, face à une " perte retrouvée». En effet, au cours de mes recherches, j'ai compris que le sentiment de la perte pour les habitants d'une ville martyre (Dresde ou Potsdam par exemple, mais aussi Rimini) peut surgir très longtemps (des années, voir des siècles) après le moment précis de cette perte. La notion de perte retrouvée n'est-elle pas issue d'une construction sociale (individuelle et collective) où se joue la distance, la récompense, la punition, la culpabilité, la fierté, l'appartenance, la revanche... des sentiments identitaires à l'œuvre dans le patrimoine sujet?

Plus un monument a une haute valeur en tant qu'exemple unique dans I'histoire de l'architecture universelle (unicum), plus sa restauration post-bellico va tendre vers un idéal de restauration « à l'identique». On verra comment les restaurations ont oscillé entre reconstructions dite "à l'identique » (come era dove era) et restaurations plus créatives, voire "critiques».

\section{Essai de périodisation de l'activité de restauration post-bellico}

\begin{tabular}{|c|c|c|c|c|c|c|c|c|}
\hline Terrains & 1945 & 1950 & 1960 & 1970 & 1980 & 1990 & 2000 & $2014 \ldots$ \\
\hline Allemagne & & & & & & & & \\
\hline France & & & & & & & & \\
\hline Italie & & & & & & & & \\
\hline
\end{tabular}

\section{Hypothèse d'interprétation de ce qui} se joue dans ces périodes identifiées

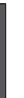

Les «Trente Glorieuses », période chaude (1945-1973) pour la restauration des monuments historiques post-bellico. C'est alors que l'essentiel se joue pour les avancées théoriques et méthodologiques, 1964 est une date clé.
19. En réalité on peut remonter infiniment dans le temps (avant 1914), et voir combien ce thème, la reconstruction post-bellico, est comme un fil d'Ariane qui permet de traverser le labyrinthe de toute l'histoire de l'architecture, les guerres et les révolutions étant, inlassablement suivies de " période chaude » de reconstruction. 
Les restaurations post-bellico peuvent être d'un très haut niveau qualitatif, car elles sont parfois conçues par de grands architectes dans les trois pays étudiés (exemple: église du Souvenir de Berlin, Alte Pinakothek de Munich, église Saint-Malo de Valognes, temple de Malatesta à Rimini). On observe de grandes avancées techniques et scientifiques, dès la fin de la période, avec la mise en place des laboratoires de recherches sur la conservation des matériaux, via la physique, la chimie, la biologie. Les laboratoires italiens et belges sont très avancés à ce sujet (ICR, Iccrom, Opificio delle Pietre Dure, IRPA à Bruxelles, etc). Les progrès sont importants dans les méthodes de relevé (stéréophotogrammétrie). Durant cette période chaude, le besoin de reconstruire comporte une dimension morale, éthique et artistique prégnante (réponse à une " perte chaude ", difficulté matérielle, pénurie, reconstruction d'un espace économique et politique européen), le corpus de cas mobilisés va démontrer cela.

\footnotetext{
La fin de la guerre froide (1973-1989) période intermédiaire ou " période froide ». Chantiers de restauration de monuments historiques en cours, mais rythme moins intense, on continue pour tenter de les achever, les chantiers de la "période chaude».
}

Complément, fin de chantiers, comme à Cologne (1985: année des églises romanes de Cologne), ou à Rimini (concours pour la reconstruction du théâtre Galli). Mon hypothèse est que l'on note dans cette période moins d'avancées sur les questions théoriques et méthodologiques alors que les progrès se font plus sur le plan des techniques de restauration des matériaux, des décors, des peintures. Pour une partie des acteurs, dans les terrains étudiés, la dimension morale et artistique des restaurations est toujours prégnante (exemple: la Glyptothèque de Munich)

Période de "la perte retrouvée ", 1989-2014, chute du mur de Berlin, unification de l'Allemagne, éclatement de I'URSS, guerre des Balkans, repli identitaire, destruction intentionnelle de sites historiques et de biens culturels au niveau mondial (Mali, Syrie, Afghanistan, etc). Reprise des chantiers de restauration post-bellico, nouvelle période chaude.

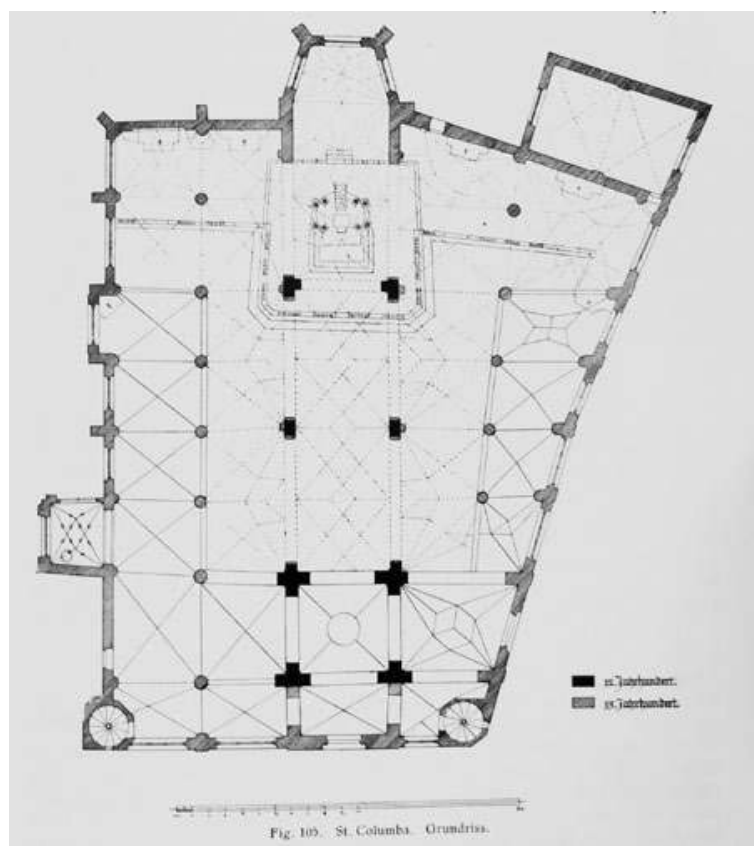

Cologne, plan de l'église de Sainte-Colombe, relevé en 1916, en haut à droite la sacristie.

Sources: Paul Clemen (dir.), Die
Kunstdenkmäler der Rheinprovinz. Die Kirchlichen Denkmäler der Stadt Köln, band, IV, Düsseldorf, Druck und Verlag, 1916 
Jusqu'en 1989 les experts de l'ex-RFA (Allemagne de I'Ouest), sont contre les reconstructions « à l'identique " des monuments détruits de l'ex-RDA (Allemagne de l'Est). Mais depuis 1990, toute l'Allemagne se lance dans une nouvelle vague de chantiers de reconstruction de monuments détruits depuis plusieurs décennies; idem pour l'ex-URSS. On reconstruit, également «à l'identique ", des monuments détruits lors des conflits récents comme à Sarajevo ou à Mostar. Les progrès techniques continuent, notamment via le développement des outils informatiques appliqués à la conservation des biens culturels (documentation, relevés 2D et 3D, scannerlaser, etc.). On constate également des progrès dans le domaine des structures (calcul aux éléments finis). On note une attention accrue pour la conservation des matériaux et des logiques constructives, pour le nettoyage des matériaux (laser). Un corpus important de textes se constitue dans le domaine technique, mais

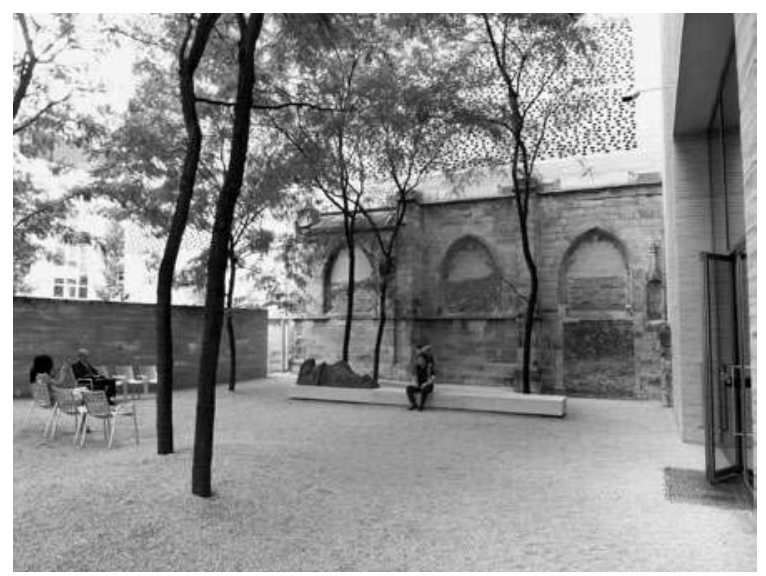

Cologne, le jardin du musée d'art sacré Kolumba. Murs en brique, zones perforées (passage de l'air et de la lumière) réintégration d'une grande lacune qui devient une nouvelle architecture.

Photo N. Detry, 2013.

également sur l'histoire et les méthodes de la restauration des monuments (avec un retour sur le passé). Les échanges internationaux se poursuivent (notamment via L'Icom et l'Icomos). J'émets l'hypothèse que pour la nouvelle période chaude, les restaurations post-bellico se jouent plus en termes politiques et économiques, comme une forme de "marketing urbain», alors que la dimension morale et artistique des restaurations tend à s'opacifier (exemple: la reconstruction du château de Berlin ou "Humboldt Forum», ou celle du palais des Ducs de Vilnius). En même temps, la dimension morale et artistique des restaurations reste présente au cas par cas, comme chez quelques acteurs de haut niveau (Bologne, ex-Oratorio dei Filippini, architecte, Pierluigi Cervellati; Berlin, le Neues Museum, architecte, David Chipperfield; Cologne, le musée Kolumba, architecte, Peter Zumthor; Géorgie, la cathédrale de Bagrati, architecte, Andrea Bruno).

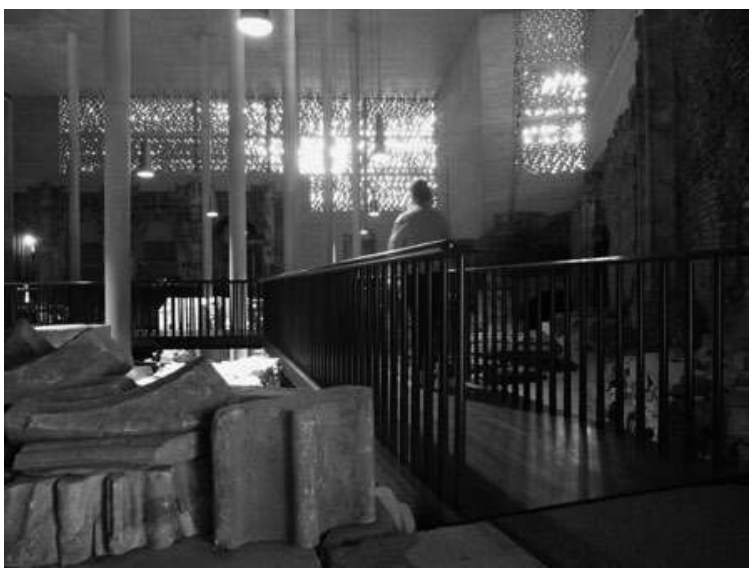

des fouilles archéologiques. Les murs perforés en briques, sont comme la réintégration d'une lacune de grande dimension. Photo N. Detry, 2013.
20. Je remercie l'architecte Sovrintendente Antonella Ranaldi, le professeur Giovanni Carbonara, le profeseur Paul Philippot, I'architecte Andrea Bruno, le professeur Gabi Dolff-Bonekämper, le professeur Francesco Bandarin, I'architecte Simone Ricca, I'architecte Jean-Louis Taupin, le professeur Michael Petzet, le professeur Gregor Hitzfeld, l'architecte Christiane Schmuckle-Mollard, I'architecte Goran Nikšić, I'architecte Boris Cindric, pour leurs conseils précieux. 


\section{Les sources et les terrains}

En parallèle avec les différents terrains étudiés, une partie de la recherche est consacrée à l'analyse d'un corpus de textes sur les méthodes et les théories de la restauration (1945-1973) avec l'émergence de la notion de restauro critico en Italie. Je m'appuie principalement sur les écrits italiens autour de la restauration critique, mais pas uniquement, car, j'ai mobilisé des témoins de plusieurs pays ${ }^{20}$. Ensuite j'examine l'application de ces "lignes guides" à un certain nombre de monuments ruinés et peu à peu restaurés ${ }^{21}$.

\section{Conclusion ouverte}

Est-il plus juste de conserver la ruine - témoin contre la barbarie des guerres - comme Saint-Alban und Gürzenich de Cologne? Comment et pourquoi, I'ex-Oratorio dei Filippini de Bologne, bombardé en 1943, a-t-il été restauré selon les principes du restauro critico en 2000? Pourquoi récupérer les pierres d'un monument détruit et créer une œuvre totalement nouvelle comme Sankt-Anna de Düren, œuvre de Rudolf Schwarz? La Alte Pinakothek de Munich et le Neues Museum de Berlin ont été restaurés, à cinquante ans de distance, dans une même approche critique et créative.

La restauration " à l'identique ", I'ultra-conservation, l'intégration de l'ancien et du nouveau, I'anastylose, la réintégration des lacunes, l'esthétique du "ruineux", le rapport matière/image, le fragment et la totalité, sont des concepts utiles qu'il faut savoir mobiliser dans une lecture critique de l'architecture patrimoniale et de ses enjeux contemporains. Je peux affirmer, comme praticien de la restauration des sites et monuments historiques, que cela reste un sujet ouvert, fluctuant, non soumis à des règles fixes. Un sujet hypersensible où toute approche doctrinaire, systémique est inappropriée mais où, en revanche, la recherche universitaire et interdisciplinaire est un complément essentiel à la pratique du métier d'architecte-restaurateur. La polémique actuelle à propos des bouddhas de Bamiyan, le montre bien. Fin janvier 2014, I'architecte italien Andrea Bruno m'a fait part de son désaccord avec le professeur Michael Petzet (expert de I'Icomos). Je venais de rencontrer celui-ci à Munich et il m'avait montré avec passion les débuts de la reconstruction par anastylose des pieds du petit bouddha de Bamiyan ${ }^{22}$. Dans un futur que nous ne pouvons qu'espérer proche, comment allonsnous aborder la reconstruction d'Alep, quand la paix va revenir? Même si le monde d'aujourd'hui semble si différent, prendre appui sur l'immense expérience de nos pères, face à l'Europe détruite en 1945, sera déjà un bon départ.

II restera toujours des questions ouvertes et sensibles: pourquoi faire une restauration différente? Comment accepter que la reconstruction ne puisse pas nécessairement rendre aux habitants, l'édifice, la maison, le monument, l'œuvre d'art, tant aimés, exactement tels qu'ils étaient avant la guerre? C'est alors que le monument apparaît dans toutes ses dimensions de patrimoine sujet, moins fait de pierres que d'images, de discours, de stratégies, de désir.
21. Nous pouvons citer comme sources principales:

1. Les monuments eux-mêmes (monument = document), recherches pour illustrer l'état avant, la destruction, la phase d'études, de concours, les chantiers de restauration, l'état actuel;

2. En bibliothèque, les articles publiés dans les revues spécialisées et dans les livres. Consultation de bibliothèques et des centres de documentations dans les pays étudiés (Allemagne, France, Italie); comme l'Icomos et l'INAH à Paris et I'Iccrom à Rome.
3. Dans les archives (archives des dessins, archives photographiques et archives documents); notamment les archives des SBAP en Italie (Soprintendenza per i Beni Architettonici e Paesaggistici), les archives de collectivités territoriales comme à Padoue; en France la MAP (médiathèque de l'Architecture et du Patrimoine) à Charenton-le-Pont.

4. Les traductions en français des textes importants de l'italien (faites par l'auteur) et de l'allemand (réalisée à la demande de l'auteur par Marieke Hofmann et Philipp Schroeter);

5. Les entretiens avec les acteurs de certains chantiers de reconstruction et avec les experts des terrains étudiés (architectes, professeurs, historiens, etc.); mais aussi avec des habitants de telle ou telle ville, entretiens avec des services de l'armée de terre, avec les forces de l'ONU envoyées durant le conflit de l'ex-Yougoslavie pour Sarajevo.

22. Voir Alessandro Martini et Ermanno Rivetti, "Unesco stops unauthorised reconstruction of Bamiyan Buddhas », lissue, $n^{\circ}$ 254, 6 février 2014; The Art Newspaper (theartnewspaper.com). 
Cologne, le nouveau

Kolumba Museum,

construit sur les ruines

de l'ancienne église

de Sainte-Colombe.

Photo N. Detry, 2013.
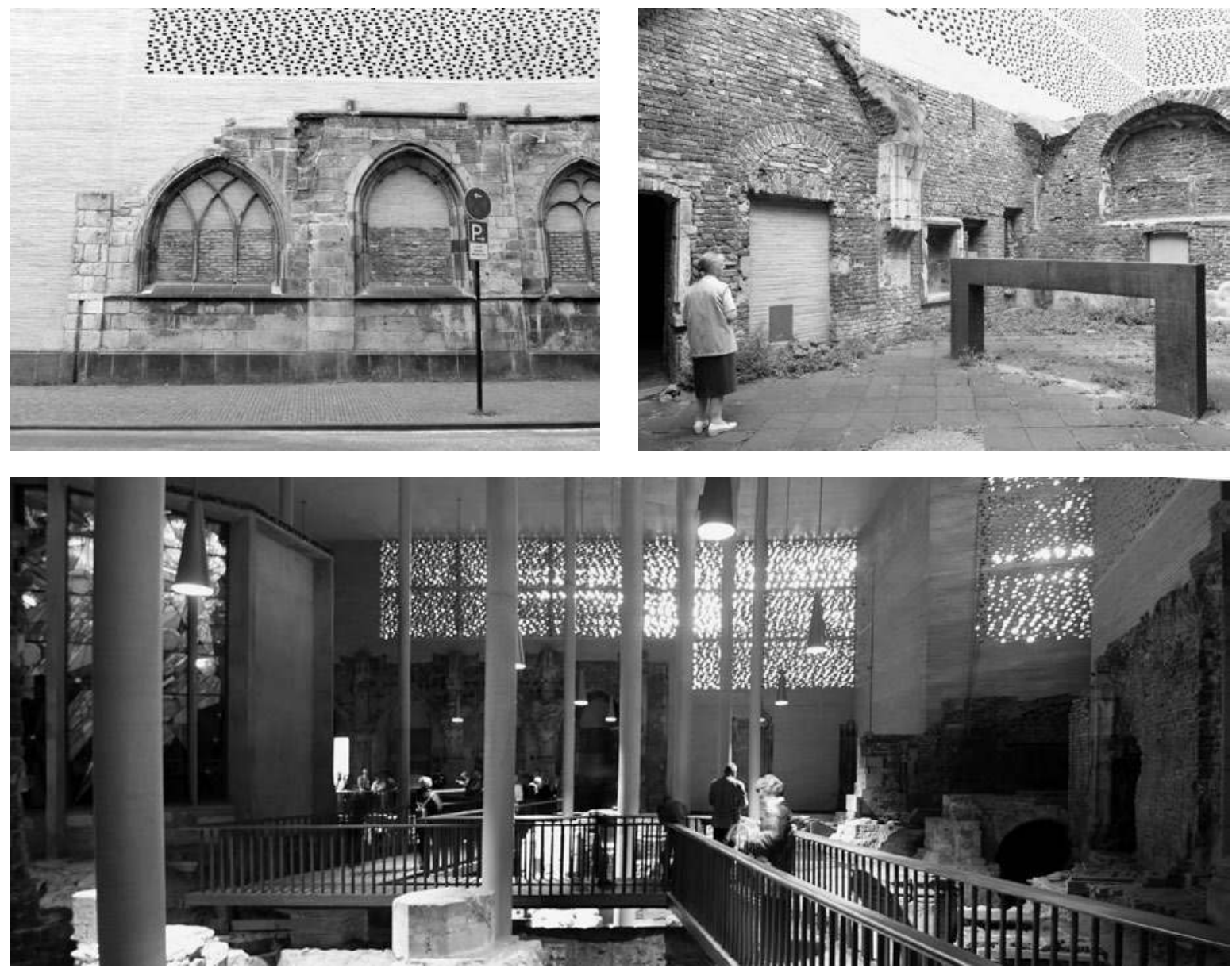

Cologne, le musée Kolumba de Peter Zumthor, la crypte archéologique avec à gauche la Chapelle de la « Madonne in des Trümmern ",
1957 (Architecte

Gottfried Böhm).

Deux architectes

lauréats du

" Pritzker Prize » se

retrouvent dans un

travail sur la ruine.

Photo N. Detry, 2013. 
J'ai choisi d'illustrer cet article avec deux exemples en Italie et en Allemagne: l'église et monastère degli Eremitani de Padoue, l'Oratorio dei Filippini à Bologne et l'ancienne église monastère de Sankt-Columba à Cologne. Pour les trois cas illustrés ici de façon synthétique, on retrouve plusieurs thématiques expliquées dans l'article:

- projets et travaux qui s'étendent sur un temps long et englobent les trois périodes identifées dans l'essai de périodisation de l'activité de restauration post-bellico (période chaude, période intermédiaire, nouvelle période chaude);

- les Eremitani de Padoue, est l'un des cas où face au traumatisme de la perte, la question de la restauration a été affrontée sur des bases théoriques renouvelées, approche qui culmine dans l'oeuvre de Cesare Brandi, Teoria del restauro (Rome, 1963);

- trois formes de remise en question des " anciennes méthodes " (Charte d'Athènes de 1931), à mettre en relation avec la « restauration critique " élaborée principalement par Roberto Pane, Renato Bonelli et Paul Philippot; approche critique qui a fait l'objet d'un compromis " onusien " dans la Charte de Venise de 1964; - projets de restauration accompagnés de polémiques (encore ouvertes à Padoue et à Cologne); et de plusieurs concours d'architecture autour des ruines de guerres;
- ces polémiques montrent que I'approche critique et créative de la restauration exige un haut niveau de culture ainsi qu'une grande capacité artistique; ce qui fait dire à certains auteurs qu'il s'agit d'une vision élitiste (voire dépassée, car élaborée il y a 50 ans) et somme toute peu compréhensible pour le grand public.

Padoue (Italie), église et monastère degli Eremitani et Museo Civico

À Padoue, l'église abbatiale degli Ermitani ( $\mathrm{XIII}^{\mathrm{e}}$-XV $\mathrm{V}^{\mathrm{e}}$ siècles) est bombardée le 11 mars 1944. La destruction de la chapelle Ovetari abritant les célèbres fresques de Andrea Mantegna (1443), déclenche une émotion qui dépasse les frontières de la péninsule; émotion non seulement chez les plus grands historiens de ce temps, mais aussi émotion populaire (perte chaude). Pourtant brisées en plusieurs milliers d'infimes fragments, la restauration réussie des fresques de Mantegna, reconstituées par I'ICR sous la direction de Cesare Brandi, force l'admiration. La convergence des méthodes entre la restauration des œuvres d'art (les peintures murales) et la restauration de l'architecture constitue un laboratoire pour l'élaboration des nouvelles méthodes. Ces méthodes sont encore valides aujourd'hui.
Durant les années 1960, la ville de Padoue, poursuit son travail de restauration des bâtiments abbatiaux. Les deux cloîtres, sont très endommagés: à la fois du fait des bombardements de 1944, et du fait d'une transformation radicale en caserne Gattamelata au $\mathrm{XIX}$ siècle (dont les plans sont attribués à Camillo Boito). La ville décide de transformer le complexe en Museo Civico degli Eremitani.

L'acteur principal de la création de ce grand musée, est l'architecte Franco Albini (1905-1977). Pourtant, en 1967, à I'unanimité, l'architecte romain Maurizio Sacripanti (1916-1996) est désigné lauréat du concours pour la construction du Museo Civico de Padoue, malgré cela, dès 1968 , le chantier est confié à Franco Albini. Retrouvé dans les archives de la ville de Padoue, le projet de Maurizio Sacripanti, était une sorte d'anticipation du centre Georges Pompidou de paris. Le futuriste Sacripanti, amant des superstructures tubulaires, est qualifié par Bruno Zevi de " un idraulico tra Giotto e Mantegna " (un plombier entre Giotto e Mantegna). Entre 1968 et 1980, le studio Albini (Albini + Franca Helg) élabore plus de 10 versions pour l'entrée du Museo Civico, un avant-corps implanté au nord de la façade de l'église. L'une des versions de cet avant-corps est bâtie vers 1970. Inachevé, il ne résiste pas aux polémiques des uns et des autres puisqu'il est détruit en 1996.

\footnotetext{
1. En 1739, elle est décrite en ces termes " architecture du Torregiani, de la plus grande beauté. Les ornements y sont répandus avec tant de goût que leur quantité n'altère point la simplicité de l'édifice ». Charles de Brosses, Lettre Familières, séjour à Bologne, lettre XII à Mr de Quintin, 19/09/1739. (rééditions, Naples, 1991, p. 385).
}

\author{
2. Pier-Luigi_Cervellati né en 1936 est un \\ professeur, architecte et urbaniste italien \\ de renommée internationale, notamment \\ pour son expérience modèle de rénovation \\ urbaine de Bologne, voir Cervellati P-L, \\ Scannavini R, De Angelis C, La nuova \\ Cultura delle Città, édition Mondadori, \\ Milano 1977.
}


On touche là un point important: la guerre, est l'un des moteurs des processus de démolition, mais n'est pas I'unique cause des démolitions. En effet, un nombre incalculable de maisons, de monuments endommagés par la guerre, sont ensuite démolis ou dynamités, car jugés, à tort ou à raison, comme gênants, non-reconstructibles, innoportuns, horribles... Ces démolitions post-bellico laissent le terrain libre pour que se poursuive la fabrique continue de la ville.

Ainsi, en 2014, I'entrée du Museo Civico de Padoue, la reconstruction du théâtre Galli de Rimini (bombardé en 1943) restent deux questions non résolues. Cette forme italienne de " perte retrouvée ", sorte d'éternel inachèvement ne doit en aucun cas être confondue avec la perte retrouvée de l'ex-Allemagne de l'Est ou des pays de l'ex-URSS, pays où les ruptures ont été très violentes, et où I'on assiste à une nouvelle vague de reconstruction dite " à l'identique" En Italie, les monuments transmis de façon continue forment (du moins ils formaient, jusqu'aux " années Berlusconi ") comme un "écosystème familier " pour la société civile italienne.

\section{Bologne (Italie), ex Oratorio di San Filippo Neri}

Bologne, subit les bombardements aériens des alliés à plusieurs reprises, I'oratoire de San Filippo Neri est touché le 29 janvier 1944. Surintendant à Bologne entre 1943 et 1959 , Alfredo Barbacci est responsable de plusieurs grands chantiers de restauration post-bellico à Bologne, dont le complexe monastique de San Francesco, la salle anatomique de l'Archiginnasio, la Loggia della Mercanzia, illustres monuments également touchés par les bombes. Inaugurée en 1733, La chapelle de San Filippo Neri est conçu par I'architecte Alfonso Torregiani, abritant des œuvres de Carlo Nessi (stucs), de Ludovico Carracci et Francesco Monti ${ }^{1}$. On trouve en Italie, du Piémont à la Sicile, des centaines de chapelles baroques cette qualité. Est-ce cela qui explique le fait qu'Alfredo Barbacci n'a pas eut la possibilité de terminer la restauration entamée dans l'immédiate après guerre? En revanche, Barbacci a eut l'intelligence de réaliser le sauvetage de l'édifice (toiture, arc triomphal en brique, colonnes en béton, maçonnerie en brique du flanc Sud). Il faudra attendre 50 ans et diverses vicissitudes (oubli, usages comme garage automobile, etc), pour que l'ex Oratorio, grâce à une fondation bancaire privée, trouve une nouvelle affectation: une salle de spectacle dédiée au théâtre et à la musique. Dès 1997, un projet global de restauration et de mise en valeur est étudié par l'architecte Pier-Luigi Cervellati ${ }^{2}$, commandité par la Fondazione del Monte; mécène de ce projet. L'œuvre de P-L Cervellati s'inscrit dans une approche critique et créative de la restauration. Le travail de Barbacci (période chaude), est laissé à l'abandon; repris 50 ans après (nouvelle période chaude), ce travail est alors " historicisé ${ }^{3}$ et doit donc être conservé comme témoin ( $1{ }^{\mathrm{er}}$ sens du mot martyr).

\section{Cologne (Allemagne),} église de Sankt-Kolumba

Fondée vers 980, l'église de SainteColombe (Sankt-Klumba ou Columba) est à l'origine l'une des nombreuses églises romanes de Cologne. Elle est remaniée et agrandie à plusieurs reprises (bas-côté ajoutés du $\mathrm{XII}^{\mathrm{e}}$ au $\mathrm{XV}^{\mathrm{e}}$ siècles, chœur vouté au $\mathrm{xvII^{ \textrm {e } }}$ siècle, intérieur baroquisés aux $\mathrm{XVII}{ }^{\mathrm{e}}$ et $x \mathrm{xIII}$ siècles). La ville de Cologne est détruite à $90 \%$ durant la Seconde Guerre mondiale. À la demande des habitants de Cologne, I'architecte Gottfried Böhm conçoit la chapelle de la "Madonna in den Trümmern " (chapelle Sainte-Marie des Ruines). Celle-ci est construite en deux temps:
3. «La parte ricostruita nel secondo dopoguerra e che testimonia la drammaticità della bomba, viene considerata " storicizzata ». Essa è un quarto della superficie delle pareti. L'ipotesi di un eventuale ripristino filologico appare impraticabile per l'artisticità del modello di riferimento. Le volte e la cupola - di consequenza - saranno ricostruite mediante un'armature lignea... L'obiettivo è quello di non cancellare il lavoro eseguito cinquant'anni fa e, a un tempo, di non alterare, accostando con pseudo ripristini o con soluzioni intermedie, ciò che restà... di un capolavoro rococò... " : dans Pier-Luigi Cervellati, «Ex-Oratorio dei Filippini, progetto di restauro, Relazione ", octobre-novembre 1997. Sources SBAP, Bo. AD, 18744. 
première phase en 1947 (mélange de création et de restauration des restes de l'édifice romano-gothique), seconde phase en 1957 avec une nouvelle construction hexagonale adossée à l'ancienne. Celle-ci est couverte par des coques légères en béton armé évoquant des voiles de tissus. Les parois laisse filtrer la lumière à travers les vitraux de Ludwig Gies 4. Le complexe de Sankt-Kulumba fait l'objet de nombreux projets et de plusieurs concours d'architecture (projet remarquable non réalisé de G. Böhm, 1948, qui anticipe les solutions réalisées à Saint-Malo de Valognes ou à la cathédrale de Coventry). Le musée d'art "Kolumba », fondé en 1853, lié au dioscèse de Cologne conserve une collection très importante d'art chrétien. L'objet de ce musée est de mettre en relation l'art ancien et contemporain afin de voir comment, les diverses périodes (sur 2000 ans d'histoire) ont abordés les grands thèmes de l'art sacré. Lauréat $d$ 'un concours international d'architecture, le projet de l'architecte suisse Peter Zumthor a été construit entre 2003 et 2007. Bâti en brique beige à la romaine, le bâtiment à la fois massif et léger de Peter Zumthor (né en 1943, Prizker Prize 2009) englobe dans son volume, les deux chapelles, "Madonna in den Trümmern " œuvres de Gottfreid Böhm (né en 1920, Prizker Prize 1986).

4. Ludwig Gies (1887-1966), artiste allemand de premier plan, plusieurs œuvres de Gies confisquées par les Nazis car considérées comme « Entartete Kunst » (exposition "d'art dégénéré » à Munich en 1937). Son aigle du Bundestag allemand, dessiné en 1953 est aujourd'hui dans le Reichstag de Berlin.
* Le présent article, est un compte rendu de ma thèse de doctorat en cours de rédaction (soutenance prévue début 2015 à l'ENSAL). Le laboratoire EVS RIVES (UMR 5600) m'accueille comme doctorant en architecture. Directeurs de thèse: Vincent Veschambre (ENSAL), Giovanni Carbonara (La Sapienza, Roma), Yves Winkin (ENS-Lyon). 